This document is the Supplementary Material of a Published Work that appeared in final form in The Journal of Molecular Spectroscopy, copyright (c) Elsevier Inc., under the citation mentioned below:

Investigation of the rotamers of 3-furfural by microwave spectroscopy (C) 2020 by Carolyn Gregory, Jennifer van Wijngaarden is licensed under CC BY-NC-ND 4.0

DOI: https://doi.org/10.1016/j.jms.2020.111374

\title{
Investigation of the Rotamers of 3-Furfural by Microwave Spectroscopy
}

Carolyn Gregory and Jennifer van Wijngaarden*

Department of Chemistry, University of Manitoba, Winnipeg, Manitoba, R3T 2N2, Canada

*Corresponding author

Email: vanwijng@cc.umanitoba.ca

Phone: (204)474-8379

Fax: (204)474-7608

Appendix I: Equilibrium (B3LYP-D3(BJ)/aug-cc-pVTZ) and literature ground state spectroscopic constants

Appendix II: Equilibrium Structures from B3LYP-D3(BJ)/aug-cc-pVTZ

Appendix III: Assigned Transitions for 3-furfural

Appendix IV: Kraitchman Coordinates $\left(r_{s}\right)$ 


\section{Appendix I: Equilibrium (B3LYP-D3(BJ)/aug-cc-pVTZ) and literature ground state spectroscopic constants}

Table S1: Equilibrium spectroscopic constants (B3LYP-D3(BJ)/aug-cc-pVTZ)(this work) and ground state constants reported in ref $13^{\text {b }}$ for 3-furfural (3-FF)

\begin{tabular}{|c|c|c|c|}
\hline & anti-3-FF & syn-3-FF ${ }^{\mathrm{a}}$ & anti-3-FF \\
\hline \multicolumn{4}{|c|}{ Rotational Constants / MHz } \\
\hline A & 8282.3 & 8223.0 & $8238.7276(16)$ \\
\hline B & 1983.0 & 1990.9 & $1976.13883(42)$ \\
\hline $\mathrm{C}$ & 1599.9 & 1602.8 & $1593.97114(34)$ \\
\hline \multicolumn{4}{|c|}{ Centrifugal Distortion Constants $/ \mathrm{kHz}$} \\
\hline$\Delta_{\jmath}$ & 0.1340 & 0.1396 & $0.13902(89)$ \\
\hline$\Delta_{\mathrm{JK}}$ & 0.5615 & 0.6123 & $0.7482(92)$ \\
\hline$\Delta_{\mathrm{K}}$ & 2.005 & 1.808 & $1.7616(89)$ \\
\hline$\delta_{\jmath}$ & 0.03049 & 0.03166 & $0.03099(17)$ \\
\hline$\delta_{K}$ & 0.7650 & 0.7882 & $0.829(14)$ \\
\hline \multicolumn{4}{|c|}{ Sextic Centrifugal Distortion Constants / Hz } \\
\hline$\Phi_{\mathrm{JK}}$ & & & $-0.001331(45)$ \\
\hline no. of lines & - & - & 239 \\
\hline $\mathrm{rms} / \mathrm{MHz}$ & - & - & 0.046 \\
\hline
\end{tabular}

\section{Appendix II: Equilibrium Structures from B3LYP-D3(BJ)/aug-cc-pVTZ}

Table S2: Z-matrix structure for anti-3-furfural.

$\begin{array}{rrrrrrr}\text { O1 } & & & & & & \\ \text { C2 } & 1 & & & 1.3476147 & & \\ \text { C3 } & 2 & 1 & & 1.3654363 & 110.4710932 & \\ \text { C4 } & 3 & 2 & 1 & 1.4381344 & 106.0111748 & 0.0015286 \\ \text { C5 } & 4 & 3 & 2 & 1.3487330 & 106.0858748 & 0.0040830 \\ \text { C6 } & 3 & 4 & 5 & 1.4563540 & 128.2295757 & -179.9963108 \\ \text { O7 } & 6 & 3 & 4 & 1.2114324 & 124.4127059 & -0.0308925 \\ \text { H8 } & 6 & 3 & 4 & 1.1072730 & 114.9065594 & 179.9711021 \\ \text { H9 } & 2 & 3 & 4 & 1.0753804 & 132.9047653 & -179.9993759 \\ \text { H10 } & 4 & 5 & 1 & 1.0750104 & 127.6398597 & 179.9960227 \\ \text { H11 } & 5 & 4 & 3 & 1.0740305 & 134.0427382 & 179.9982408\end{array}$


Table S3: Z-matrix structure for syn-3-furfural.

$\begin{array}{rlllllr}\text { O1 } & & & & & \\ \text { C2 } & 1 & & & 1.3471198 & \\ \text { C3 } & 2 & 1 & & 1.3658406 & 110.3111591 & \\ \text { C4 } & 3 & 2 & 1 & 1.4384051 & 105.8951122 & 0.0039864 \\ \text { C5 } & 4 & 3 & 2 & 1.3505592 & 106.2834220 & -0.0155861 \\ \text { C6 } & 3 & 4 & 5 & 1.4603413 & 127.3597456 & 179.9909142 \\ \text { O7 } & 6 & 3 & 4 & 1.2113517 & 124.7679229 & 179.9553334 \\ \text { H8 } & 6 & 3 & 4 & 1.1072665 & 114.6949431 & -0.0382961 \\ \text { H9 } & 2 & 3 & 4 & 1.0742074 & 132.5302331 & -179.9945191 \\ \text { H10 } & 4 & 5 & 1 & 1.0761759 & 126.6848154 & -179.9894812 \\ \text { H11 } & 5 & 4 & 3 & 1.0738432 & 134.1316705 & -179.9939883\end{array}$

\section{Appendix III: Assigned Transitions for 3-furfural}

Table S4: anti-3-furfural (parent)

\begin{tabular}{ccccccccc}
\hline $\mathrm{J}^{\prime}$ & $\mathrm{K}_{\mathrm{a}}{ }^{\prime}$ & $\mathrm{K}_{\mathrm{c}}{ }^{\prime}$ & $\mathrm{J}^{\prime \prime}$ & $\mathrm{K}_{\mathrm{a}}{ }^{\prime \prime}$ & $\mathrm{K}_{\mathrm{c}^{\prime \prime}}$ & $v_{\text {obs }} / \mathrm{MHz}$ & $v_{\text {calc }} / \mathrm{MHz}$ & $v_{\text {obs }- \text { calc }} / \mathrm{MHz}$ \\
\hline 1 & 1 & 0 & 1 & 0 & 1 & 6644.7541 & 6644.7539 & 0.0002 \\
2 & 1 & 2 & 1 & 1 & 1 & 6758.0521 & 6758.0521 & 0.0000 \\
2 & 1 & 1 & 2 & 0 & 2 & 7043.8761 & 7043.8761 & 0.0000 \\
2 & 0 & 2 & 1 & 0 & 1 & 7123.2573 & 7123.2573 & 0.0000 \\
2 & 1 & 1 & 1 & 1 & 0 & 7522.3800 & 7522.3795 & 0.0005 \\
3 & 1 & 2 & 3 & 0 & 3 & 7673.8936 & 7673.8935 & 0.0001 \\
6 & 1 & 5 & 6 & 1 & 6 & 7986.0578 & 7986.0578 & 0.0000 \\
4 & 1 & 3 & 4 & 0 & 4 & 8570.4687 & 8570.4686 & 0.0000 \\
4 & 0 & 4 & 3 & 1 & 3 & 8731.3918 & 8731.3923 & -0.0004 \\
5 & 1 & 4 & 5 & 0 & 5 & 9776.1467 & 9776.1466 & 0.0001 \\
2 & 2 & 0 & 3 & 1 & 3 & 9824.4945 & 9824.4954 & -0.0009 \\
1 & 1 & 1 & 0 & 0 & 0 & 9832.7004 & 9832.7005 & 0.0000 \\
3 & 1 & 3 & 2 & 1 & 2 & 10126.6965 & 10126.6968 & -0.0002 \\
7 & 1 & 6 & 7 & 1 & 7 & 10602.7699 & 10602.7697 & 0.0001 \\
3 & 0 & 3 & 2 & 0 & 2 & 10642.6953 & 10642.6958 & -0.0005 \\
3 & 2 & 2 & 2 & 2 & 1 & 10710.3033 & 10710.3021 & 0.0011 \\
3 & 2 & 1 & 2 & 2 & 0 & 10777.9267 & 10777.9260 & 0.0007 \\
3 & 1 & 2 & 2 & 1 & 1 & 11272.7128 & 11272.7132 & -0.0004 \\
6 & 1 & 5 & 6 & 0 & 6 & 11333.3833 & 11333.3832 & 0.0000 \\
5 & 0 & 5 & 4 & 1 & 4 & 12768.7909 & 12768.7909 & 0.0000 \\
2 & 1 & 2 & 1 & 0 & 1 & 13020.6415 & 13020.6415 & 0.0000 \\
7 & 1 & 6 & 7 & 0 & 7 & 13274.7926 & 13274.7927 & 0.0000 \\
4 & 1 & 4 & 3 & 1 & 3 & 13483.5318 & 13483.5320 & -0.0002 \\
8 & 1 & 7 & 8 & 1 & 8 & 13544.7076 & 13544.7075 & 0.0000 \\
4 & 0 & 4 & 3 & 0 & 3 & 14112.7773 & 14112.7774 & -0.0001
\end{tabular}




\begin{tabular}{rrrrrrrrr}
4 & 2 & 3 & 3 & 2 & 2 & 14267.1902 & 14267.1904 & -0.0001 \\
4 & 3 & 2 & 3 & 3 & 1 & 14312.9091 & 14312.9090 & 0.0001 \\
4 & 3 & 1 & 3 & 3 & 0 & 14315.7316 & 14315.7316 & 0.0000 \\
4 & 2 & 2 & 3 & 2 & 1 & 14434.8124 & 14434.8127 & -0.0002 \\
4 & 1 & 3 & 3 & 1 & 2 & 15009.3525 & 15009.3525 & 0.0000 \\
4 & 1 & 3 & 3 & 1 & 2 & 15009.3525 & 15009.3525 & 0.0000 \\
8 & 1 & 7 & 8 & 0 & 8 & 15613.1183 & 15613.1185 & -0.0002 \\
3 & 1 & 3 & 2 & 0 & 2 & 16024.0806 & 16024.0809 & -0.0003 \\
8 & 2 & 6 & 8 & 1 & 7 & 16147.0072 & 16147.0074 & -0.0001 \\
7 & 2 & 5 & 7 & 1 & 6 & 16276.4602 & 16276.4603 & 0.0000 \\
6 & 2 & 4 & 6 & 1 & 5 & 16637.0613 & 16637.0612 & 0.0001 \\
6 & 0 & 6 & 5 & 1 & 5 & 16803.7130 & 16803.7126 & 0.0004 \\
5 & 1 & 5 & 4 & 1 & 4 & 16825.6685 & 16825.6684 & 0.0000 \\
5 & 2 & 3 & 5 & 1 & 4 & 17151.0144 & 17151.0139 & 0.0004 \\
5 & 0 & 5 & 4 & 0 & 4 & 17520.9309 & 17520.9307 & 0.0002 \\
4 & 2 & 2 & 4 & 1 & 3 & 17735.3737 & 17735.3732 & 0.0005 \\
5 & 2 & 4 & 4 & 2 & 3 & 17812.7788 & 17812.7788 & 0.0000 \\
5 & 4 & 2 & 4 & 4 & 1 & 17888.0507 & 17888.0508 & -0.0001 \\
5 & 4 & 1 & 4 & 4 & 0 & 17888.1374 & 17888.1375 & -0.0001 \\
5 & 3 & 3 & 4 & 3 & 2 & 17903.9267 & 17903.9268 & -0.0001 \\
5 & 3 & 2 & 4 & 3 & 1 & 17913.7789 & 17913.7792 & -0.0003 \\
5 & 2 & 3 & 4 & 2 & 2 & 18142.2492 & 18142.2493 & -0.0001 \\
3 & 2 & 1 & 3 & 1 & 2 & 18309.9133 & 18309.9130 & 0.0003 \\
5 & 1 & 4 & 4 & 1 & 3 & 18726.6089 & 18726.6086 & 0.0002 \\
2 & 2 & 0 & 2 & 1 & 1 & 18804.6997 & 18804.7002 & -0.0004 \\
4 & 1 & 4 & 3 & 0 & 3 & 18864.9169 & 18864.9171 & -0.0002 \\
\hline & & & & & & & & \\
\hline
\end{tabular}

Table S5: anti-3-furfural $\left({ }^{18} \mathrm{O} 1\right)$

\begin{tabular}{ccccccccc}
\hline $\mathrm{J}^{\prime}$ & $\mathrm{K}_{\mathrm{a}}^{\prime}$ & $\mathrm{K}_{\mathrm{c}}{ }^{\prime}$ & $\mathrm{J}^{\prime \prime}$ & $\mathrm{K}_{\mathrm{a}}^{\prime \prime}$ & $\mathrm{K}_{\mathrm{c}^{\prime \prime}}$ & $v_{\text {obs }} / \mathrm{MHz}$ & $v_{\text {calc }} / \mathrm{MHz}$ & $v_{\text {obs }- \text { calc }} / \mathrm{MHz}$ \\
\hline 2 & 1 & 2 & 1 & 1 & 1 & 6587.8418 & 6587.8436 & -0.0018 \\
1 & 1 & 0 & 1 & 0 & 1 & 6627.0603 & 6627.0603 & 0.0000 \\
2 & 0 & 2 & 1 & 0 & 1 & 6937.3526 & 6937.3550 & -0.0023 \\
2 & 1 & 1 & 2 & 0 & 2 & 7007.5562 & 7007.5559 & 0.0003 \\
2 & 1 & 1 & 1 & 1 & 0 & 7317.8502 & 7317.8506 & -0.0004 \\
3 & 1 & 2 & 3 & 0 & 3 & 7606.9522 & 7606.9521 & 0.0000 \\
4 & 0 & 4 & 3 & 1 & 3 & 8335.0386 & 8335.0381 & 0.0004 \\
4 & 1 & 3 & 4 & 0 & 4 & 8457.9837 & 8457.9844 & -0.0007 \\
5 & 1 & 4 & 5 & 0 & 5 & 9600.0512 & 9600.0518 & -0.0006 \\
1 & 1 & 1 & 0 & 0 & 0 & 9738.4834 & 9738.4827 & 0.0007 \\
3 & 1 & 3 & 2 & 1 & 2 & 9872.2725 & 9872.2730 & -0.0005 \\
3 & 0 & 3 & 2 & 0 & 2 & 10367.4725 & 10367.4726 & 0.0000 \\
3 & 2 & 2 & 2 & 2 & 1 & 10429.2518 & 10429.2499 & 0.0018 \\
3 & 2 & 1 & 2 & 2 & 0 & 10491.0458 & 10491.0449 & 0.0009
\end{tabular}




\begin{tabular}{rrrrrrrrr}
3 & 1 & 2 & 2 & 1 & 1 & 10966.8686 & 10966.8687 & -0.0001 \\
6 & 1 & 5 & 6 & 0 & 6 & 11073.2467 & 11073.2465 & 0.0001 \\
5 & 0 & 5 & 4 & 1 & 4 & 12269.4450 & 12269.4447 & 0.0003 \\
2 & 1 & 2 & 1 & 0 & 1 & 12849.9013 & 12849.8997 & 0.0015 \\
4 & 1 & 4 & 3 & 1 & 3 & 13145.8780 & 13145.8784 & -0.0003 \\
4 & 0 & 4 & 3 & 0 & 3 & 13752.3831 & 13752.3833 & -0.0001 \\
4 & 2 & 3 & 3 & 2 & 2 & 13893.5979 & 13893.5971 & 0.0007 \\
4 & 3 & 2 & 3 & 3 & 1 & 13935.4134 & 13935.4129 & 0.0005 \\
4 & 3 & 1 & 3 & 3 & 0 & 13937.8783 & 13937.8793 & -0.0009 \\
4 & 2 & 2 & 3 & 2 & 1 & 14046.8814 & 14046.8808 & 0.0006 \\
4 & 1 & 3 & 3 & 1 & 2 & 14603.4160 & 14603.4156 & 0.0004 \\
3 & 1 & 3 & 2 & 0 & 2 & 15784.8179 & 15784.8178 & 0.0000 \\
6 & 0 & 6 & 5 & 1 & 5 & 16207.9758 & 16207.9755 & 0.0003 \\
5 & 1 & 5 & 4 & 1 & 4 & 16405.9684 & 16405.9689 & -0.0005 \\
5 & 0 & 5 & 4 & 0 & 4 & 17080.2847 & 17080.2849 & -0.0002 \\
5 & 2 & 4 & 4 & 2 & 3 & 17347.6197 & 17347.6201 & -0.0004 \\
5 & 3 & 3 & 4 & 3 & 2 & 17431.0718 & 17431.0705 & 0.0013 \\
5 & 3 & 2 & 4 & 3 & 1 & 17439.6806 & 17439.6817 & -0.0011 \\
5 & 2 & 3 & 4 & 2 & 2 & 17649.3347 & 17649.3352 & -0.0004 \\
5 & 1 & 4 & 4 & 1 & 3 & 18222.3521 & 18222.3524 & -0.0002 \\
4 & 1 & 4 & 3 & 0 & 3 & 18563.2231 & 18563.2236 & -0.0004 \\
\hline
\end{tabular}

Table S6: anti-3-furfural $\left({ }^{13} \mathrm{C} 2\right)$

\begin{tabular}{ccccccccc}
\hline $\mathrm{J}^{\prime}$ & $\mathrm{K}_{\mathrm{a}}^{\prime}$ & $\mathrm{K}_{\mathrm{c}}{ }^{\prime}$ & $\mathrm{J}^{\prime \prime}$ & $\mathrm{K}_{\mathrm{a}}^{\prime \prime}$ & $\mathrm{K}_{\mathrm{c}^{\prime \prime}}$ & $v_{\text {obs }} / \mathrm{MHz}$ & $v_{\text {calc }} / \mathrm{MHz}$ & $v_{\text {obs }- \text { calc }} / \mathrm{MHz}$ \\
\hline 1 & 1 & 0 & 1 & 0 & 1 & 6496.3379 & 6496.3402 & -0.0023 \\
2 & 1 & 2 & 1 & 1 & 1 & 6727.9796 & 6727.9803 & -0.0006 \\
2 & 1 & 1 & 2 & 0 & 2 & 6900.7961 & 6900.7984 & -0.0022 \\
2 & 0 & 2 & 1 & 0 & 1 & 7096.8861 & 7096.8863 & -0.0002 \\
2 & 1 & 1 & 1 & 1 & 0 & 7501.3452 & 7501.3445 & 0.0007 \\
3 & 1 & 2 & 3 & 0 & 3 & 7540.3033 & 7540.3042 & -0.0008 \\
4 & 1 & 3 & 4 & 0 & 4 & 8452.0888 & 8452.0881 & 0.0006 \\
4 & 0 & 4 & 3 & 1 & 3 & 8833.0132 & 8833.0139 & -0.0006 \\
1 & 1 & 1 & 0 & 0 & 0 & 9666.9928 & 9666.9918 & 0.0010 \\
5 & 1 & 4 & 5 & 0 & 5 & 9680.2309 & 9680.2306 & 0.0003 \\
3 & 1 & 3 & 2 & 1 & 2 & 10081.0975 & 10081.0973 & 0.0001 \\
3 & 0 & 3 & 2 & 0 & 2 & 10601.1236 & 10601.1232 & 0.0004 \\
3 & 2 & 2 & 2 & 2 & 1 & 10671.9694 & 10671.9721 & -0.0027 \\
3 & 1 & 2 & 2 & 1 & 1 & 11240.6307 & 11240.6290 & 0.0017 \\
6 & 1 & 5 & 6 & 0 & 6 & 11267.9767 & 11267.9773 & -0.0005 \\
2 & 1 & 2 & 1 & 0 & 1 & 12837.6394 & 12837.6378 & 0.0015 \\
5 & 0 & 5 & 4 & 1 & 4 & 12853.0119 & 12853.0118 & 0.0001 \\
4 & 1 & 4 & 3 & 1 & 3 & 13421.8697 & 13421.8700 & -0.0003 \\
4 & 0 & 4 & 3 & 0 & 3 & 14053.7393 & 14053.7396 & -0.0002
\end{tabular}




\begin{tabular}{rrrrrrrrr}
4 & 2 & 3 & 3 & 2 & 2 & 14215.4485 & 14215.4481 & 0.0003 \\
4 & 3 & 1 & 3 & 3 & 0 & 14266.3867 & 14266.3868 & -0.0001 \\
4 & 2 & 2 & 3 & 2 & 1 & 14391.0033 & 14391.0022 & 0.0011 \\
4 & 1 & 3 & 3 & 1 & 2 & 14965.5245 & 14965.5235 & 0.0010 \\
3 & 1 & 3 & 2 & 0 & 2 & 15821.8493 & 15821.8488 & 0.0004 \\
5 & 2 & 3 & 5 & 1 & 4 & 16696.1266 & 16696.1263 & 0.0003 \\
5 & 1 & 5 & 4 & 1 & 4 & 16747.3175 & 16747.3182 & -0.0006 \\
6 & 0 & 6 & 5 & 1 & 5 & 16864.9726 & 16864.9712 & 0.0013 \\
4 & 2 & 2 & 4 & 1 & 3 & 17274.4102 & 17274.4107 & -0.0004 \\
5 & 0 & 5 & 4 & 0 & 4 & 17441.8668 & 17441.8678 & -0.0010 \\
5 & 2 & 4 & 4 & 2 & 3 & 17747.0819 & 17747.0824 & -0.0005 \\
5 & 3 & 3 & 4 & 3 & 2 & 17842.4496 & 17842.4501 & -0.0004 \\
3 & 2 & 1 & 3 & 1 & 2 & 17848.9334 & 17848.9320 & 0.0013 \\
5 & 3 & 2 & 4 & 3 & 1 & 17853.1480 & 17853.1467 & 0.0012 \\
5 & 2 & 3 & 4 & 2 & 2 & 18091.7254 & 18091.7259 & -0.0004 \\
4 & 1 & 4 & 3 & 0 & 3 & 18642.5945 & 18642.5956 & -0.0011 \\
5 & 1 & 4 & 4 & 1 & 3 & 18670.0095 & 18670.0103 & -0.0007 \\
\hline
\end{tabular}

Table S7: anti-3-furfural $\left({ }^{13} \mathrm{C} 3\right)$

\begin{tabular}{ccccccccc}
\hline $\mathrm{J}^{\prime}$ & $\mathrm{K}_{\mathrm{a}}{ }^{\prime}$ & $\mathrm{K}_{\mathrm{c}}{ }^{\prime}$ & $\mathrm{J}^{\prime \prime}$ & $\mathrm{K}_{\mathrm{a}}^{\prime \prime}$ & $\mathrm{K}_{\mathrm{c}^{\prime \prime}}$ & $v_{\text {obs }} / \mathrm{MHz}$ & $v_{\text {calc }} / \mathrm{MHz}$ & $v_{\text {obs }- \text { calc }} / \mathrm{MHz}$ \\
\hline 1 & 1 & 0 & 1 & 0 & 1 & 6640.8219 & 6640.8228 & -0.0009 \\
2 & 1 & 2 & 1 & 1 & 1 & 6756.1043 & 6756.1052 & -0.0008 \\
2 & 1 & 1 & 2 & 0 & 2 & 7039.9397 & 7039.9398 & 0.0000 \\
2 & 0 & 2 & 1 & 0 & 1 & 7121.2884 & 7121.2871 & 0.0013 \\
2 & 1 & 1 & 1 & 1 & 0 & 7520.4041 & 7520.4041 & 0.0000 \\
3 & 1 & 2 & 3 & 0 & 3 & 7669.9658 & 7669.9657 & 0.0000 \\
4 & 1 & 3 & 4 & 0 & 4 & 8566.5798 & 8566.5798 & 0.0000 \\
5 & 1 & 4 & 5 & 0 & 5 & 9772.3419 & 9772.3420 & 0.0000 \\
1 & 1 & 1 & 0 & 0 & 0 & 9827.8037 & 9827.8031 & 0.0006 \\
3 & 1 & 3 & 2 & 1 & 2 & 10123.7707 & 10123.7711 & -0.0004 \\
7 & 1 & 6 & 6 & 2 & 5 & 10583.8530 & 10583.8528 & 0.0001 \\
3 & 0 & 3 & 2 & 0 & 2 & 10639.7179 & 10639.7183 & -0.0003 \\
3 & 2 & 2 & 2 & 2 & 1 & 10707.3609 & 10707.3606 & 0.0002 \\
3 & 2 & 1 & 2 & 2 & 0 & 10775.0210 & 10775.0204 & 0.0006 \\
3 & 1 & 2 & 2 & 1 & 1 & 11269.7441 & 11269.7442 & -0.0001 \\
6 & 1 & 5 & 6 & 0 & 6 & 11329.7123 & 11329.7118 & 0.0005 \\
5 & 0 & 5 & 4 & 1 & 4 & 12767.4657 & 12767.4657 & 0.0000 \\
2 & 1 & 2 & 1 & 0 & 1 & 13014.7777 & 13014.7778 & 0.0000 \\
7 & 1 & 6 & 7 & 0 & 7 & 13271.2851 & 13271.2853 & -0.0002 \\
4 & 1 & 4 & 3 & 1 & 3 & 13479.6217 & 13479.6219 & -0.0002 \\
4 & 0 & 4 & 3 & 0 & 3 & 14108.7680 & 14108.7681 & 0.0000 \\
4 & 2 & 3 & 3 & 2 & 2 & 14263.2621 & 14263.2615 & 0.0006 \\
4 & 3 & 2 & 3 & 3 & 1 & 14309.0049 & 14309.0043 & 0.0005
\end{tabular}




\begin{tabular}{rrrrrrrrr}
4 & 3 & 1 & 3 & 3 & 0 & 14311.8298 & 14311.8300 & -0.0002 \\
4 & 2 & 2 & 3 & 2 & 1 & 14430.9709 & 14430.9711 & -0.0001 \\
4 & 1 & 3 & 3 & 1 & 2 & 15005.3817 & 15005.3821 & -0.0004 \\
3 & 1 & 3 & 2 & 0 & 2 & 16017.2618 & 16017.2619 & 0.0000 \\
7 & 2 & 5 & 7 & 1 & 6 & 16265.9873 & 16265.9877 & -0.0004 \\
6 & 2 & 4 & 6 & 1 & 5 & 16626.0912 & 16626.0907 & 0.0004 \\
6 & 0 & 6 & 5 & 1 & 5 & 16801.1425 & 16801.1423 & 0.0002 \\
5 & 1 & 5 & 4 & 1 & 4 & 16820.7674 & 16820.7674 & 0.0000 \\
5 & 2 & 3 & 5 & 1 & 4 & 17139.6904 & 17139.6903 & 0.0001 \\
5 & 0 & 5 & 4 & 0 & 4 & 17515.8631 & 17515.8631 & 0.0000 \\
4 & 2 & 2 & 4 & 1 & 3 & 17723.8241 & 17723.8232 & 0.0009 \\
5 & 2 & 4 & 4 & 2 & 3 & 17807.8564 & 17807.8565 & 0.0000 \\
5 & 3 & 3 & 4 & 3 & 2 & 17899.0510 & 17899.0513 & -0.0003 \\
5 & 3 & 2 & 4 & 3 & 1 & 17908.9140 & 17908.9146 & -0.0006 \\
5 & 2 & 3 & 4 & 2 & 2 & 18137.4923 & 18137.4924 & 0.0000 \\
3 & 2 & 1 & 3 & 1 & 2 & 18298.2347 & 18298.2343 & 0.0004 \\
5 & 1 & 4 & 4 & 1 & 3 & 18721.6253 & 18721.6253 & 0.0000 \\
2 & 2 & 0 & 2 & 1 & 1 & 18792.9566 & 18792.9581 & -0.0014 \\
4 & 1 & 4 & 3 & 0 & 3 & 18857.1659 & 18857.1655 & 0.0004 \\
\hline
\end{tabular}

Table S8: anti-3-furfural $\left({ }^{13} \mathrm{C} 4\right)$

\begin{tabular}{ccccccccc}
\hline $\mathrm{J}^{\prime}$ & $\mathrm{K}_{\mathrm{a}}^{\prime}$ & $\mathrm{K}_{\mathrm{c}}{ }^{\prime}$ & $\mathrm{J}^{\prime \prime}$ & $\mathrm{K}_{\mathrm{a}}^{\prime \prime}$ & $\mathrm{K}_{\mathrm{c}^{\prime \prime}}$ & $v_{\text {obs }} / \mathrm{MHz}$ & $v_{\text {calc }} / \mathrm{MHz}$ & $v_{\text {obs }- \text { calc }} / \mathrm{MHz}$ \\
\hline 1 & 1 & 0 & 1 & 0 & 1 & 6490.4258 & 6490.4256 & 0.0001 \\
2 & 1 & 2 & 1 & 1 & 1 & 6737.1841 & 6737.1840 & 0.0001 \\
2 & 1 & 1 & 2 & 0 & 2 & 6896.3326 & 6896.3321 & 0.0004 \\
2 & 0 & 2 & 1 & 0 & 1 & 7107.2600 & 7107.2601 & -0.0001 \\
2 & 1 & 1 & 1 & 1 & 0 & 7513.1669 & 7513.1666 & 0.0002 \\
3 & 1 & 2 & 3 & 0 & 3 & 7538.2627 & 7538.2637 & -0.0009 \\
4 & 1 & 3 & 4 & 0 & 4 & 8453.7220 & 8453.7221 & -0.0001 \\
4 & 0 & 4 & 3 & 1 & 3 & 8862.5728 & 8862.5734 & -0.0005 \\
1 & 1 & 1 & 0 & 0 & 0 & 9665.0261 & 9665.0244 & 0.0017 \\
5 & 1 & 4 & 5 & 0 & 5 & 9687.0644 & 9687.0652 & -0.0007 \\
3 & 1 & 3 & 2 & 1 & 2 & 10094.8179 & 10094.8186 & -0.0006 \\
3 & 0 & 3 & 2 & 0 & 2 & 10616.3389 & 10616.3398 & -0.0008 \\
3 & 2 & 2 & 2 & 2 & 1 & 10687.7403 & 10687.7409 & -0.0006 \\
3 & 2 & 1 & 2 & 2 & 0 & 10759.1600 & 10759.1604 & -0.0003 \\
3 & 1 & 2 & 2 & 1 & 1 & 11258.2723 & 11258.2713 & 0.0009 \\
6 & 1 & 5 & 6 & 0 & 6 & 11281.7134 & 11281.7136 & -0.0001 \\
2 & 1 & 2 & 1 & 0 & 1 & 12839.6187 & 12839.6175 & 0.0011 \\
7 & 1 & 6 & 7 & 0 & 7 & 13269.4742 & 13269.4745 & -0.0002 \\
4 & 1 & 4 & 3 & 1 & 3 & 13440.0155 & 13440.0163 & -0.0008 \\
4 & 0 & 4 & 3 & 0 & 3 & 14073.4094 & 14073.4096 & -0.0001 \\
4 & 2 & 3 & 3 & 2 & 2 & 14236.3640 & 14236.3642 & -0.0001
\end{tabular}




\begin{tabular}{rrrrrrrrr}
4 & 3 & 2 & 3 & 3 & 1 & 14284.6061 & 14284.6056 & 0.0005 \\
4 & 3 & 1 & 3 & 3 & 0 & 14287.7088 & 14287.7083 & 0.0005 \\
4 & 2 & 2 & 3 & 2 & 1 & 14413.2731 & 14413.2721 & 0.0010 \\
4 & 1 & 3 & 3 & 1 & 2 & 14988.8690 & 14988.8681 & 0.0009 \\
3 & 1 & 3 & 2 & 0 & 2 & 15827.1752 & 15827.1760 & -0.0008 \\
6 & 2 & 4 & 6 & 1 & 5 & 16174.0470 & 16174.0477 & -0.0006 \\
5 & 1 & 5 & 4 & 1 & 4 & 16769.7791 & 16769.7799 & -0.0007 \\
4 & 2 & 2 & 4 & 1 & 3 & 17250.4851 & 17250.4859 & -0.0007 \\
5 & 0 & 5 & 4 & 0 & 4 & 17465.5411 & 17465.5416 & -0.0005 \\
5 & 2 & 4 & 4 & 2 & 3 & 17773.0520 & 17773.0527 & -0.0007 \\
3 & 2 & 1 & 3 & 1 & 2 & 17826.0832 & 17826.0818 & 0.0014 \\
5 & 3 & 3 & 4 & 3 & 2 & 17869.1435 & 17869.1433 & 0.0001 \\
5 & 3 & 2 & 4 & 3 & 1 & 17879.9713 & 17879.9710 & 0.0003 \\
5 & 2 & 3 & 4 & 2 & 2 & 18120.3003 & 18120.2994 & 0.0009 \\
4 & 1 & 4 & 3 & 0 & 3 & 18650.8515 & 18650.8526 & -0.0010 \\
5 & 1 & 4 & 4 & 1 & 3 & 18698.8854 & 18698.8847 & 0.0007 \\
\hline
\end{tabular}

Table S9: anti-3-furfural $\left({ }^{13} \mathrm{C} 5\right)$

\begin{tabular}{ccccccccc}
\hline $\mathrm{J}^{\prime}$ & $\mathrm{K}_{\mathrm{a}}{ }^{\prime}$ & $\mathrm{K}_{\mathrm{c}}{ }^{\prime}$ & $\mathrm{J}^{\prime \prime}$ & $\mathrm{K}_{\mathrm{a}}{ }^{\prime \prime}$ & $\mathrm{K}_{\mathrm{c}^{\prime \prime}}$ & $v_{\text {obs }} / \mathrm{MHz}$ & $v_{\text {calc }} / \mathrm{MHz}$ & $v_{\text {obs }- \text { calc }} / \mathrm{MHz}$ \\
\hline 1 & 1 & 0 & 1 & 0 & 1 & 6562.8672 & 6562.8674 & -0.0002 \\
2 & 1 & 2 & 1 & 1 & 1 & 6683.6798 & 6683.6786 & 0.0011 \\
2 & 1 & 1 & 2 & 0 & 2 & 6958.1190 & 6958.1191 & 0.0000 \\
2 & 0 & 2 & 1 & 0 & 1 & 7045.2576 & 7045.2576 & 0.0000 \\
2 & 1 & 1 & 1 & 1 & 0 & 7440.5094 & 7440.5092 & 0.0001 \\
3 & 1 & 2 & 3 & 0 & 3 & 7582.1028 & 7582.1027 & 0.0000 \\
4 & 0 & 4 & 3 & 1 & 3 & 8645.6716 & 8645.6704 & 0.0012 \\
5 & 1 & 4 & 5 & 0 & 5 & 9664.6529 & 9664.6535 & -0.0005 \\
1 & 1 & 1 & 0 & 0 & 0 & 9715.5017 & 9715.5013 & 0.0004 \\
3 & 1 & 3 & 2 & 1 & 2 & 10015.2105 & 10015.2120 & -0.0015 \\
3 & 0 & 3 & 2 & 0 & 2 & 10526.0006 & 10526.0018 & -0.0011 \\
3 & 2 & 2 & 2 & 2 & 1 & 10593.1211 & 10593.1205 & 0.0006 \\
3 & 2 & 1 & 2 & 2 & 0 & 10660.2560 & 10660.2552 & 0.0007 \\
6 & 1 & 5 & 6 & 0 & 6 & 11207.4873 & 11207.4866 & 0.0007 \\
5 & 0 & 5 & 4 & 1 & 4 & 12638.6981 & 12638.6986 & -0.0005 \\
2 & 1 & 2 & 1 & 0 & 1 & 12868.1310 & 12868.1300 & 0.0010 \\
7 & 1 & 6 & 7 & 0 & 7 & 13130.9303 & 13130.9306 & -0.0002 \\
4 & 1 & 4 & 3 & 1 & 3 & 13335.0230 & 13335.0235 & -0.0004 \\
4 & 0 & 4 & 3 & 0 & 3 & 13957.7525 & 13957.7530 & -0.0005 \\
4 & 2 & 3 & 3 & 2 & 2 & 14111.0432 & 14111.0436 & -0.0003 \\
4 & 3 & 2 & 3 & 3 & 1 & 14156.4294 & 14156.4311 & -0.0016 \\
4 & 3 & 1 & 3 & 3 & 0 & 14159.2433 & 14159.2406 & 0.0026 \\
4 & 2 & 2 & 3 & 2 & 1 & 14277.4460 & 14277.4461 & 0.0000 \\
4 & 1 & 3 & 3 & 1 & 2 & 14845.8632 & 14845.8636 & -0.0003
\end{tabular}




\begin{tabular}{rrrrrrrrr}
3 & 1 & 3 & 2 & 0 & 2 & 15838.0842 & 15838.0844 & -0.0001 \\
8 & 2 & 6 & 8 & 1 & 7 & 15946.7778 & 15946.7777 & 0.0001 \\
7 & 2 & 5 & 7 & 1 & 6 & 16072.0572 & 16072.0574 & -0.0001 \\
6 & 2 & 4 & 6 & 1 & 5 & 16426.9225 & 16426.9233 & -0.0007 \\
6 & 0 & 6 & 5 & 1 & 5 & 16628.8765 & 16628.8753 & 0.0012 \\
5 & 1 & 5 & 4 & 1 & 4 & 16640.2475 & 16640.2480 & -0.0004 \\
5 & 2 & 3 & 5 & 1 & 4 & 16934.3196 & 16934.3188 & 0.0007 \\
5 & 0 & 5 & 4 & 0 & 4 & 17328.0515 & 17328.0518 & -0.0002 \\
4 & 2 & 2 & 4 & 1 & 3 & 17512.0192 & 17512.0179 & 0.0013 \\
5 & 2 & 4 & 4 & 2 & 3 & 17617.7482 & 17617.7484 & -0.0002 \\
5 & 3 & 3 & 4 & 3 & 2 & 17708.2289 & 17708.2294 & -0.0005 \\
5 & 3 & 2 & 4 & 3 & 1 & 17718.0360 & 17718.0362 & -0.0001 \\
5 & 2 & 3 & 4 & 2 & 2 & 17944.7928 & 17944.7929 & 0.0000 \\
3 & 2 & 1 & 3 & 1 & 2 & 18080.4353 & 18080.4353 & 0.0000 \\
5 & 1 & 4 & 4 & 1 & 3 & 18522.4917 & 18522.4920 & -0.0002 \\
2 & 2 & 0 & 2 & 1 & 1 & 18570.1643 & 18570.1655 & -0.0012 \\
4 & 1 & 4 & 3 & 0 & 3 & 18647.1070 & 18647.1062 & 0.0008 \\
\hline
\end{tabular}

Table S10: anti-3-furfural $\left({ }^{13} \mathrm{C} 6\right)$

\begin{tabular}{ccccccccc}
\hline $\mathrm{J}^{\prime}$ & $\mathrm{K}_{\mathrm{a}}^{\prime}$ & $\mathrm{K}_{\mathrm{c}}^{\prime}$ & $\mathrm{J}^{\prime \prime}$ & $\mathrm{K}_{\mathrm{a}}^{\prime \prime}$ & $\mathrm{K}_{\mathrm{c}^{\prime \prime}}$ & $v_{\text {obs }} / \mathrm{MHz}$ & $v_{\text {calc }} / \mathrm{MHz}$ & $v_{\text {obs }-c a l c} / \mathrm{MHz}$ \\
\hline 2 & 1 & 2 & 1 & 1 & 1 & 6689.0156 & 6689.0159 & -0.0002 \\
2 & 1 & 1 & 2 & 0 & 2 & 7019.4710 & 7019.4738 & -0.0027 \\
2 & 0 & 2 & 1 & 0 & 1 & 7048.2711 & 7048.2716 & -0.0005 \\
2 & 1 & 1 & 1 & 1 & 0 & 7440.3759 & 7440.3755 & 0.0003 \\
3 & 1 & 2 & 3 & 0 & 3 & 7637.9853 & 7637.9868 & -0.0015 \\
4 & 1 & 3 & 4 & 0 & 4 & 8517.5046 & 8517.5053 & -0.0006 \\
4 & 0 & 4 & 3 & 1 & 3 & 8582.6042 & 8582.6044 & -0.0002 \\
5 & 1 & 4 & 5 & 0 & 5 & 9699.4283 & 9699.4278 & 0.0005 \\
1 & 1 & 1 & 0 & 0 & 0 & 9784.0400 & 9784.0403 & -0.0003 \\
3 & 1 & 3 & 2 & 1 & 2 & 10023.4652 & 10023.4663 & -0.0010 \\
3 & 0 & 3 & 2 & 0 & 2 & 10531.5395 & 10531.5402 & -0.0006 \\
3 & 2 & 2 & 2 & 2 & 1 & 10597.0226 & 10597.0224 & 0.0002 \\
3 & 2 & 1 & 2 & 2 & 0 & 10662.5236 & 10662.5221 & 0.0014 \\
3 & 1 & 2 & 2 & 1 & 1 & 11150.0540 & 11150.0533 & 0.0007 \\
6 & 1 & 5 & 6 & 0 & 6 & 11225.3469 & 11225.3474 & -0.0004 \\
5 & 0 & 5 & 4 & 1 & 4 & 12578.3905 & 12578.3903 & 0.0001 \\
2 & 1 & 2 & 1 & 0 & 1 & 12940.7055 & 12940.7052 & 0.0003 \\
4 & 1 & 4 & 3 & 1 & 3 & 13346.4653 & 13346.4664 & -0.0011 \\
4 & 0 & 4 & 3 & 0 & 3 & 13966.9630 & 13966.9642 & -0.0011 \\
4 & 2 & 3 & 3 & 2 & 2 & 14116.5685 & 14116.5675 & 0.0010 \\
4 & 3 & 1 & 3 & 3 & 0 & 14163.5584 & 14163.5569 & 0.0015 \\
4 & 2 & 2 & 3 & 2 & 1 & 14278.9661 & 14278.9647 & 0.0013 \\
4 & 1 & 3 & 3 & 1 & 2 & 14846.4828 & 14846.4826 & 0.0002
\end{tabular}




\begin{tabular}{rrrrrrrrr}
3 & 1 & 3 & 2 & 0 & 2 & 15915.8994 & 15915.9000 & -0.0005 \\
6 & 0 & 6 & 5 & 1 & 5 & 16573.9906 & 16573.9906 & 0.0000 \\
6 & 2 & 4 & 6 & 1 & 5 & 16623.0252 & 16623.0232 & 0.0020 \\
5 & 1 & 5 & 4 & 1 & 4 & 16655.1982 & 16655.1993 & -0.0010 \\
5 & 2 & 3 & 5 & 1 & 4 & 17136.7666 & 17136.7668 & -0.0002 \\
5 & 0 & 5 & 4 & 0 & 4 & 17342.2508 & 17342.2523 & -0.0014 \\
5 & 2 & 4 & 4 & 2 & 3 & 17625.1693 & 17625.1683 & 0.0010 \\
5 & 2 & 3 & 4 & 2 & 2 & 17944.5264 & 17944.5251 & 0.0013 \\
3 & 2 & 1 & 3 & 1 & 2 & 18283.9350 & 18283.9344 & 0.0006 \\
5 & 1 & 4 & 4 & 1 & 3 & 18524.1746 & 18524.1749 & -0.0002 \\
4 & 1 & 4 & 3 & 0 & 3 & 18730.8247 & 18730.8262 & -0.0015 \\
\hline
\end{tabular}

Table S11: anti-3-furfural $\left({ }^{18} \mathrm{O} 7\right)$

\begin{tabular}{ccccccccc}
\hline $\mathrm{J}^{\prime}$ & $\mathrm{K}_{\mathrm{a}}{ }^{\prime}$ & $\mathrm{K}_{\mathrm{c}}{ }^{\prime}$ & $\mathrm{J}^{\prime \prime}$ & $\mathrm{K}_{\mathrm{a}}{ }^{\prime \prime}$ & $\mathrm{K}_{\mathrm{c}^{\prime \prime}}$ & $v_{\text {obs }} / \mathrm{MHz}$ & $v_{\text {calc }} / \mathrm{MHz}$ & $v_{\text {obs }- \text { calc }} / \mathrm{MHz}$ \\
\hline 2 & 1 & 2 & 1 & 1 & 1 & 6468.3584 & 6468.3576 & 0.0008 \\
1 & 1 & 0 & 1 & 0 & 1 & 6681.4130 & 6681.4122 & 0.0007 \\
2 & 0 & 2 & 1 & 0 & 1 & 6804.2372 & 6804.2377 & -0.0004 \\
2 & 1 & 1 & 2 & 0 & 2 & 7045.5113 & 7045.5109 & 0.0004 \\
2 & 1 & 1 & 1 & 1 & 0 & 7168.3358 & 7168.3363 & -0.0004 \\
3 & 1 & 2 & 3 & 0 & 3 & 7617.7796 & 7617.7801 & -0.0004 \\
4 & 0 & 4 & 3 & 1 & 3 & 7978.7162 & 7978.7168 & -0.0006 \\
4 & 1 & 3 & 4 & 0 & 4 & 8428.1844 & 8428.1845 & -0.0001 \\
5 & 1 & 4 & 5 & 0 & 5 & 9513.1251 & 9513.1238 & 0.0012 \\
3 & 1 & 3 & 2 & 1 & 2 & 9693.8825 & 9693.8818 & 0.0007 \\
1 & 1 & 1 & 0 & 0 & 0 & 9740.5981 & 9740.5985 & -0.0004 \\
3 & 0 & 3 & 2 & 0 & 2 & 10171.2212 & 10171.2211 & 0.0000 \\
3 & 2 & 1 & 2 & 2 & 0 & 10283.7945 & 10283.7957 & -0.0012 \\
3 & 1 & 2 & 2 & 1 & 1 & 10743.4906 & 10743.4904 & 0.0002 \\
6 & 1 & 5 & 6 & 0 & 6 & 10910.3961 & 10910.3965 & -0.0004 \\
5 & 0 & 5 & 4 & 1 & 4 & 11839.4800 & 11839.4807 & -0.0007 \\
2 & 1 & 2 & 1 & 0 & 1 & 12799.7793 & 12799.7797 & -0.0003 \\
4 & 1 & 4 & 3 & 1 & 3 & 12909.5116 & 12909.5115 & 0.0001 \\
4 & 0 & 4 & 3 & 0 & 3 & 13496.9195 & 13496.9195 & 0.0000 \\
4 & 2 & 3 & 3 & 2 & 2 & 13625.6739 & 13625.6737 & 0.0001 \\
4 & 3 & 2 & 3 & 3 & 1 & 13663.8089 & 13663.8096 & -0.0007 \\
4 & 3 & 1 & 3 & 3 & 0 & 13665.9434 & 13665.9431 & 0.0003 \\
4 & 2 & 2 & 3 & 2 & 1 & 13765.4207 & 13765.4204 & 0.0003 \\
4 & 1 & 3 & 3 & 1 & 2 & 14307.3243 & 14307.3239 & 0.0003 \\
3 & 1 & 3 & 2 & 0 & 2 & 15689.4240 & 15689.4239 & 0.0000 \\
6 & 0 & 6 & 5 & 1 & 5 & 15711.2718 & 15711.2719 & -0.0001 \\
5 & 1 & 5 & 4 & 1 & 4 & 16112.7439 & 16112.7438 & 0.0001 \\
5 & 0 & 5 & 4 & 0 & 4 & 16770.2754 & 16770.2754 & 0.0000 \\
6 & 2 & 4 & 6 & 1 & 5 & 16921.2929 & 16921.2924 & 0.0005 \\
& & & & & & & &
\end{tabular}




\begin{tabular}{rrrrrrrrr}
5 & 2 & 4 & 4 & 2 & 3 & 17014.4415 & 17014.4412 & 0.0002 \\
5 & 3 & 3 & 4 & 3 & 2 & 17090.6297 & 17090.6294 & 0.0002 \\
5 & 3 & 2 & 4 & 3 & 1 & 17098.0798 & 17098.0801 & -0.0003 \\
5 & 2 & 3 & 4 & 2 & 2 & 17289.9243 & 17289.9245 & -0.0002 \\
5 & 1 & 4 & 4 & 1 & 3 & 17855.2151 & 17855.2147 & 0.0004 \\
4 & 2 & 2 & 4 & 1 & 3 & 18006.7500 & 18006.7515 & -0.0014 \\
4 & 1 & 4 & 3 & 0 & 3 & 18427.7150 & 18427.7142 & 0.0007 \\
\hline
\end{tabular}

Table S12: syn-3-furfural (parent)

\begin{tabular}{|c|c|c|c|c|c|c|c|c|}
\hline$J^{\prime}$ & $\mathrm{K}_{\mathrm{a}}^{\prime}$ & $\mathrm{K}_{\mathrm{c}}^{\prime}$ & $\mathrm{J}^{\prime \prime}$ & $\mathrm{K}_{\mathrm{a}}^{\prime \prime}$ & $\mathrm{K}_{\mathrm{c}^{\prime \prime}}$ & $v_{o b s} / \mathrm{MHz}$ & $v_{\text {calc }} / \mathrm{MHz}$ & $v_{\text {obs-calc }} / \mathrm{MHz}$ \\
\hline 1 & 1 & 0 & 1 & 0 & 1 & 6560.3808 & 6560.3805 & 0.0003 \\
\hline 2 & 1 & 2 & 1 & 1 & 1 & 6781.7133 & 6781.7134 & -0.0001 \\
\hline 3 & 2 & 1 & 4 & 1 & 4 & 6834.8320 & 6834.8313 & 0.0007 \\
\hline 2 & 1 & 1 & 2 & 0 & 2 & 6967.0812 & 6967.0809 & 0.0003 \\
\hline 2 & 0 & 2 & 1 & 0 & 1 & 7152.8070 & 7152.8072 & -0.0002 \\
\hline 2 & 2 & 1 & 3 & 1 & 2 & 7186.5608 & 7186.5619 & -0.0011 \\
\hline 2 & 1 & 1 & 1 & 1 & 0 & 7559.5076 & 7559.5077 & -0.0001 \\
\hline 3 & 1 & 2 & 3 & 0 & 3 & 7610.0021 & 7610.0020 & 0.0001 \\
\hline 4 & 1 & 3 & 4 & 0 & 4 & 8526.4478 & 8526.4476 & 0.0002 \\
\hline 4 & 0 & 4 & 3 & 1 & 3 & 8888.2179 & 8888.2180 & -0.0001 \\
\hline 2 & 2 & 0 & 3 & 1 & 3 & 9537.2368 & 9537.2371 & -0.0003 \\
\hline 1 & 1 & 1 & 0 & 0 & 0 & 9756.7908 & 9756.7908 & 0.0000 \\
\hline 5 & 1 & 4 & 5 & 0 & 5 & 9760.6255 & 9760.6258 & -0.0003 \\
\hline 3 & 1 & 3 & 2 & 1 & 2 & 10161.6791 & 10161.6792 & 0.0000 \\
\hline 5 & 3 & 2 & 6 & 2 & 5 & 10566.4285 & 10566.4282 & 0.0002 \\
\hline 3 & 0 & 3 & 2 & 0 & 2 & 10684.9355 & 10684.9353 & 0.0001 \\
\hline 3 & 2 & 2 & 2 & 2 & 1 & 10755.8964 & 10755.8962 & 0.0002 \\
\hline 3 & 2 & 1 & 2 & 2 & 0 & 10826.8720 & 10826.8714 & 0.0005 \\
\hline 7 & 1 & 6 & 6 & 2 & 5 & 11021.5922 & 11021.5924 & -0.0001 \\
\hline 3 & 1 & 2 & 2 & 1 & 1 & 11327.8564 & 11327.8564 & 0.0000 \\
\hline 6 & 1 & 5 & 6 & 0 & 6 & 11355.9992 & 11355.9989 & 0.0003 \\
\hline 5 & 0 & 5 & 4 & 1 & 4 & 12940.0688 & 12940.0690 & -0.0002 \\
\hline 2 & 1 & 2 & 1 & 0 & 1 & 12953.1959 & 12953.1959 & 0.0000 \\
\hline 7 & 1 & 6 & 7 & 0 & 7 & 13344.7521 & 13344.7521 & 0.0000 \\
\hline 4 & 3 & 2 & 5 & 2 & 3 & 13391.6052 & 13391.6045 & 0.0007 \\
\hline 4 & 1 & 4 & 3 & 1 & 3 & 13529.2772 & 13529.2773 & 0.0000 \\
\hline 4 & 3 & 1 & 5 & 2 & 4 & 14005.0323 & 14005.0315 & 0.0008 \\
\hline 4 & 0 & 4 & 3 & 0 & 3 & 14165.3504 & 14165.3506 & -0.0001 \\
\hline 4 & 2 & 3 & 3 & 2 & 2 & 14327.3240 & 14327.3242 & -0.0001 \\
\hline 4 & 3 & 2 & 3 & 3 & 1 & 14375.2818 & 14375.2819 & -0.0001 \\
\hline 4 & 3 & 1 & 3 & 3 & 0 & 14378.3385 & 14378.3386 & -0.0001 \\
\hline 4 & 2 & 2 & 3 & 2 & 1 & 14503.1585 & 14503.1587 & -0.0002 \\
\hline 4 & 1 & 3 & 3 & 1 & 2 & 15081.7959 & 15081.7962 & -0.0002 \\
\hline
\end{tabular}




\begin{tabular}{ccccccccc}
8 & 1 & 7 & 8 & 0 & 8 & 15737.2981 & 15737.2978 & 0.0002 \\
8 & 1 & 7 & 7 & 2 & 6 & 15879.0383 & 15879.0378 & 0.0004 \\
8 & 2 & 6 & 8 & 1 & 7 & 15929.2716 & 15929.2712 & 0.0004 \\
3 & 1 & 3 & 2 & 0 & 2 & 15962.0681 & 15962.0678 & 0.0003 \\
7 & 2 & 5 & 7 & 1 & 6 & 16025.2903 & 16025.2902 & 0.0000 \\
9 & 2 & 7 & 9 & 1 & 8 & 16145.3876 & 16145.3873 & 0.0002 \\
10 & 2 & 8 & 10 & 1 & 9 & 16730.1446 & 16730.1470 & -0.0023 \\
5 & 1 & 5 & 4 & 1 & 4 & 16881.5173 & 16881.5172 & 0.0000 \\
6 & 0 & 6 & 5 & 1 & 5 & 16984.5015 & 16984.5013 & 0.0002 \\
3 & 3 & 1 & 4 & 2 & 2 & 17248.4567 & 17248.4581 & -0.0013 \\
4 & 2 & 2 & 4 & 1 & 3 & 17452.6022 & 17452.6016 & 0.0005 \\
3 & 3 & 0 & 4 & 2 & 3 & 17513.5832 & 17513.5837 & -0.0004 \\
5 & 0 & 5 & 4 & 0 & 4 & 17581.1284 & 17581.1283 & 0.0001 \\
11 & 2 & 9 & 11 & 1 & 10 & 17729.7443 & 17729.7430 & 0.0012 \\
5 & 2 & 4 & 4 & 2 & 3 & 17886.8908 & 17886.8908 & 0.0000 \\
5 & 3 & 3 & 4 & 3 & 2 & 17982.4309 & 17982.4302 & 0.0007 \\
5 & 3 & 2 & 4 & 3 & 1 & 17993.0977 & 17993.0979 & -0.0001 \\
3 & 2 & 1 & 3 & 1 & 2 & 18031.2396 & 18031.2391 & 0.0004 \\
5 & 2 & 3 & 4 & 2 & 2 & 18232.1351 & 18232.1355 & -0.0004 \\
9 & 1 & 8 & 9 & 0 & 9 & 18515.8353 & 18515.8357 & -0.0003 \\
2 & 2 & 0 & 2 & 1 & 1 & 18532.2235 & 18532.2241 & -0.0005 \\
4 & 1 & 4 & 3 & 0 & 3 & 18806.4100 & 18806.4098 & 0.0002 \\
5 & 1 & 4 & 4 & 1 & 3 & 18815.3065 & 18815.3066 & 0.0000 \\
\hline & & & & & & & & \\
\hline
\end{tabular}

Table S13: syn-3-furfural $\left({ }^{13} \mathrm{C} 2\right)$

\begin{tabular}{ccccccccc}
\hline $\mathrm{J}^{\prime}$ & $\mathrm{K}_{\mathrm{a}}{ }^{\prime}$ & $\mathrm{K}_{\mathrm{c}}{ }^{\prime}$ & $\mathrm{J}^{\prime \prime}$ & $\mathrm{K}_{\mathrm{a}}{ }^{\prime \prime}$ & $\mathrm{K}_{\mathrm{c}^{\prime \prime}}$ & $v_{\text {obs }} / \mathrm{MHz}$ & $v_{\text {calc }} / \mathrm{MHz}$ & $v_{\text {obs }- \text { calc }} / \mathrm{MHz}$ \\
\hline 2 & 1 & 2 & 1 & 1 & 1 & 6764.8049 & 6764.8049 & 0.0000 \\
2 & 0 & 2 & 1 & 0 & 1 & 7139.7819 & 7139.7830 & -0.0011 \\
3 & 1 & 2 & 3 & 0 & 3 & 7503.6091 & 7503.6099 & -0.0008 \\
2 & 1 & 1 & 1 & 1 & 0 & 7551.9511 & 7551.9507 & 0.0004 \\
3 & 1 & 3 & 2 & 1 & 2 & 10135.8391 & 10135.8400 & -0.0008 \\
3 & 0 & 3 & 2 & 0 & 2 & 10663.4479 & 10663.4475 & 0.0004 \\
3 & 2 & 2 & 2 & 2 & 1 & 10737.5462 & 10737.5461 & 0.0001 \\
3 & 1 & 2 & 2 & 1 & 1 & 11316.0055 & 11316.0052 & 0.0003 \\
2 & 1 & 2 & 1 & 0 & 1 & 12810.1176 & 12810.1158 & 0.0018 \\
5 & 0 & 5 & 4 & 1 & 4 & 13032.3852 & 13032.3847 & 0.0004 \\
4 & 1 & 4 & 3 & 1 & 3 & 13493.9899 & 13493.9902 & -0.0002 \\
4 & 0 & 4 & 3 & 0 & 3 & 14133.2131 & 14133.2139 & -0.0007 \\
4 & 2 & 3 & 3 & 2 & 2 & 14302.2412 & 14302.2409 & 0.0002 \\
4 & 2 & 2 & 3 & 2 & 1 & 14485.7469 & 14485.7460 & 0.0008 \\
4 & 1 & 3 & 3 & 1 & 2 & 15064.9752 & 15064.9755 & -0.0002 \\
3 & 1 & 3 & 2 & 0 & 2 & 15806.1727 & 15806.1728 & 0.0000 \\
5 & 1 & 5 & 4 & 1 & 4 & 16836.1781 & 16836.1783 & -0.0002
\end{tabular}




\begin{tabular}{rrrrrrrrr}
6 & 0 & 6 & 5 & 1 & 5 & 17061.7030 & 17061.7024 & 0.0005 \\
5 & 0 & 5 & 4 & 0 & 4 & 17535.8857 & 17535.8863 & -0.0005 \\
5 & 2 & 4 & 4 & 2 & 3 & 17854.5495 & 17854.5492 & 0.0002 \\
5 & 2 & 3 & 4 & 2 & 2 & 18214.4437 & 18214.4441 & -0.0003 \\
5 & 1 & 4 & 4 & 1 & 3 & 18792.5197 & 18792.5203 & -0.0005 \\
\hline
\end{tabular}

Table S14: syn-3-furfural $\left({ }^{13} \mathrm{C} 3\right)$

\begin{tabular}{ccccccccc}
\hline $\mathrm{J}^{\prime}$ & $\mathrm{K}_{\mathrm{a}}^{\prime}$ & $\mathrm{K}_{\mathrm{c}}{ }^{\prime}$ & $\mathrm{J}^{\prime \prime}$ & $\mathrm{K}_{\mathrm{a}}^{\prime \prime}$ & $\mathrm{K}_{\mathrm{c}^{\prime \prime}}$ & $v_{\text {obs }} / \mathrm{MHz}$ & $v_{\text {calc }} / \mathrm{MHz}$ & $v_{\text {obs }- \text { calc }} / \mathrm{MHz}$ \\
\hline 2 & 1 & 2 & 1 & 1 & 1 & 6779.5380 & 6779.5381 & -0.0002 \\
2 & 1 & 1 & 2 & 0 & 2 & 6960.7150 & 6960.7157 & -0.0003 \\
2 & 0 & 2 & 1 & 0 & 1 & 7150.7050 & 7150.7051 & 0.0003 \\
2 & 1 & 1 & 1 & 1 & 0 & 7557.5340 & 7557.5339 & 0.0003 \\
3 & 1 & 2 & 3 & 0 & 3 & 7603.8790 & 7603.8791 & -0.0005 \\
4 & 1 & 3 & 4 & 0 & 4 & 8520.7330 & 8520.7330 & -0.0003 \\
4 & 0 & 4 & 3 & 1 & 3 & 8890.5280 & 8890.5289 & -0.0007 \\
3 & 1 & 3 & 2 & 1 & 2 & 10158.4000 & 10158.3998 & 0.0000 \\
3 & 0 & 3 & 2 & 0 & 2 & 10681.7100 & 10681.7144 & 0.0002 \\
3 & 2 & 2 & 2 & 2 & 1 & 10752.7800 & 10752.7848 & -0.0002 \\
3 & 2 & 1 & 2 & 2 & 0 & 10823.8700 & 10823.8690 & -0.0014 \\
3 & 1 & 2 & 2 & 1 & 1 & 11324.8800 & 11324.8778 & 0.0003 \\
6 & 1 & 5 & 6 & 0 & 6 & 11351.7700 & 11351.7662 & 0.0008 \\
5 & 0 & 5 & 4 & 1 & 4 & 12941.0900 & 12941.0878 & 0.0009 \\
2 & 1 & 2 & 1 & 0 & 1 & 12944.4300 & 12944.4264 & 0.0003 \\
7 & 1 & 6 & 7 & 0 & 7 & 13341.5800 & 13341.5770 & -0.0004 \\
4 & 1 & 4 & 3 & 1 & 3 & 13524.8800 & 13524.8761 & -0.0005 \\
4 & 0 & 4 & 3 & 0 & 3 & 14160.9400 & 14160.9355 & -0.0003 \\
4 & 2 & 3 & 3 & 2 & 2 & 14323.1500 & 14323.1543 & 0.0003 \\
4 & 2 & 2 & 3 & 2 & 1 & 14499.2600 & 14499.2551 & 0.0019 \\
4 & 1 & 3 & 3 & 1 & 2 & 15077.7900 & 15077.7894 & -0.0009 \\
3 & 1 & 3 & 2 & 0 & 2 & 15952.1200 & 15952.1210 & 0.0006 \\
5 & 1 & 5 & 4 & 1 & 4 & 16875.9700 & 16875.9738 & -0.0001 \\
5 & 0 & 5 & 4 & 0 & 4 & 17575.4300 & 17575.4351 & -0.0001 \\
5 & 2 & 4 & 4 & 2 & 3 & 17881.6400 & 17881.6445 & -0.0003 \\
5 & 2 & 3 & 4 & 2 & 2 & 18227.4000 & 18227.3962 & 0.0000 \\
4 & 1 & 4 & 3 & 0 & 3 & 18795.2800 & 18795.2827 & 0.0000 \\
5 & 1 & 4 & 4 & 1 & 3 & 18810.2400 & 18810.2366 & 0.0000 \\
\hline
\end{tabular}

Table S15: syn-3-furfural $\left({ }^{13} \mathrm{C} 4\right)$

\begin{tabular}{ccccccccc}
\hline $\mathrm{J}^{\prime}$ & $\mathrm{K}_{\mathrm{a}}{ }^{\prime}$ & $\mathrm{K}_{\mathrm{c}}{ }^{\prime}$ & $\mathrm{J}^{\prime \prime}$ & $\mathrm{K}_{\mathrm{a}}{ }^{\prime \prime}$ & $\mathrm{K}_{\mathrm{c}^{\prime \prime}}$ & $v_{\text {obs }} / \mathrm{MHz}$ & $v_{\text {calc }} / \mathrm{MHz}$ & $v_{\text {obs }- \text { calc }} / \mathrm{MHz}$ \\
\hline 2 & 1 & 2 & 1 & 1 & 1 & 6745.9462 & 6745.9455 & 0.0007 \\
2 & 1 & 1 & 2 & 0 & 2 & 6801.2756 & 6801.2776 & -0.0020 \\
2 & 0 & 2 & 1 & 0 & 1 & 7121.4105 & 7121.4102 & 0.0002
\end{tabular}




\begin{tabular}{rrrrrrrrr}
3 & 1 & 2 & 3 & 0 & 3 & 7455.6251 & 7455.6259 & -0.0008 \\
2 & 1 & 1 & 1 & 1 & 0 & 7534.5069 & 7534.5061 & 0.0008 \\
4 & 1 & 3 & 4 & 0 & 4 & 8390.4514 & 8390.4515 & -0.0001 \\
3 & 1 & 3 & 2 & 1 & 2 & 10107.4185 & 10107.4191 & -0.0006 \\
3 & 0 & 3 & 2 & 0 & 2 & 10635.3471 & 10635.3463 & 0.0007 \\
3 & 2 & 1 & 2 & 2 & 0 & 10785.3049 & 10785.3072 & -0.0022 \\
3 & 1 & 2 & 2 & 1 & 1 & 11289.6964 & 11289.6946 & 0.0018 \\
2 & 1 & 2 & 1 & 0 & 1 & 12739.8469 & 12739.8462 & 0.0006 \\
5 & 0 & 5 & 4 & 1 & 4 & 13034.8798 & 13034.8791 & 0.0006 \\
4 & 1 & 4 & 3 & 1 & 3 & 13455.8660 & 13455.8662 & -0.0002 \\
4 & 0 & 4 & 3 & 0 & 3 & 14094.7844 & 14094.7835 & 0.0008 \\
4 & 2 & 3 & 3 & 2 & 2 & 14265.7685 & 14265.7681 & 0.0003 \\
4 & 2 & 2 & 3 & 2 & 1 & 14451.3997 & 14451.4002 & -0.0005 \\
4 & 1 & 3 & 3 & 1 & 2 & 15029.6105 & 15029.6091 & 0.0014 \\
3 & 1 & 3 & 2 & 0 & 2 & 15725.8533 & 15725.8551 & -0.0017 \\
5 & 1 & 5 & 4 & 1 & 4 & 16788.1883 & 16788.1883 & 0.0000 \\
4 & 2 & 2 & 4 & 1 & 3 & 16917.8953 & 16917.8946 & 0.0007 \\
6 & 0 & 6 & 5 & 1 & 5 & 17051.2670 & 17051.2663 & 0.0007 \\
5 & 0 & 5 & 4 & 0 & 4 & 17486.4707 & 17486.4706 & 0.0000 \\
3 & 2 & 1 & 3 & 1 & 2 & 17496.1047 & 17496.1035 & 0.0012 \\
5 & 2 & 4 & 4 & 2 & 3 & 17808.6835 & 17808.6847 & -0.0012 \\
5 & 2 & 3 & 4 & 2 & 2 & 18172.6138 & 18172.6147 & -0.0008 \\
4 & 1 & 4 & 3 & 0 & 3 & 18546.3744 & 18546.3750 & -0.0006 \\
5 & 1 & 4 & 4 & 1 & 3 & 18747.8161 & 18747.8163 & -0.0002 \\
\hline
\end{tabular}

Table S16: syn-3-furfural $\left({ }^{13} \mathrm{C} 5\right)$

\begin{tabular}{ccccccccc}
\hline $\mathrm{J}^{\prime}$ & $\mathrm{K}_{\mathrm{a}}^{\prime}$ & $\mathrm{K}_{\mathrm{c}}^{\prime}$ & $\mathrm{J}^{\prime \prime}$ & $\mathrm{K}_{\mathrm{a}}^{\prime \prime}$ & $\mathrm{K}_{\mathrm{c}^{\prime \prime}}$ & $v_{\text {obs }} / \mathrm{MHz}$ & $v_{\text {calc }} / \mathrm{MHz}$ & $v_{\text {obs }- \text { calc }} / \mathrm{MHz}$ \\
\hline 2 & 1 & 1 & 2 & 0 & 2 & 6948.6139 & 6948.6145 & -0.0005 \\
2 & 0 & 2 & 1 & 0 & 1 & 7056.0648 & 7056.0642 & 0.0006 \\
3 & 1 & 2 & 3 & 0 & 3 & 7575.1808 & 7575.1799 & 0.0009 \\
4 & 1 & 3 & 4 & 0 & 4 & 8467.2228 & 8467.2217 & 0.0010 \\
4 & 0 & 4 & 3 & 1 & 3 & 8681.2811 & 8681.2824 & -0.0013 \\
5 & 1 & 4 & 5 & 0 & 5 & 9667.2527 & 9667.2541 & -0.0014 \\
3 & 1 & 3 & 2 & 1 & 2 & 10029.4918 & 10029.4912 & 0.0005 \\
3 & 0 & 3 & 2 & 0 & 2 & 10541.8316 & 10541.8307 & 0.0009 \\
3 & 2 & 2 & 2 & 2 & 1 & 10609.5643 & 10609.5633 & 0.0010 \\
3 & 1 & 2 & 2 & 1 & 1 & 11168.3963 & 11168.3960 & 0.0002 \\
6 & 1 & 5 & 6 & 0 & 6 & 11217.5424 & 11217.5419 & 0.0005 \\
5 & 0 & 5 & 4 & 1 & 4 & 12679.9460 & 12679.9451 & 0.0008 \\
2 & 1 & 2 & 1 & 0 & 1 & 12865.2931 & 12865.2936 & -0.0005 \\
7 & 1 & 6 & 7 & 0 & 7 & 13150.2568 & 13150.2566 & 0.0001 \\
4 & 1 & 4 & 3 & 1 & 3 & 13353.8990 & 13353.8980 & 0.0009 \\
4 & 0 & 4 & 3 & 0 & 3 & 13978.1737 & 13978.1724 & 0.0013
\end{tabular}




\begin{tabular}{rrrrrrrrr}
4 & 2 & 3 & 3 & 2 & 2 & 14132.8463 & 14132.8470 & -0.0006 \\
4 & 2 & 2 & 3 & 2 & 1 & 14300.7499 & 14300.7512 & -0.0012 \\
4 & 1 & 3 & 3 & 1 & 2 & 14870.2131 & 14870.2143 & -0.0012 \\
3 & 1 & 3 & 2 & 0 & 2 & 15838.7208 & 15838.7207 & 0.0000 \\
5 & 1 & 5 & 4 & 1 & 4 & 16663.5960 & 16663.5963 & -0.0003 \\
6 & 0 & 6 & 5 & 1 & 5 & 16674.9080 & 16674.9070 & 0.0009 \\
5 & 0 & 5 & 4 & 0 & 4 & 17352.5608 & 17352.5608 & 0.0000 \\
5 & 2 & 4 & 4 & 2 & 3 & 17644.8095 & 17644.8100 & -0.0005 \\
5 & 2 & 3 & 4 & 2 & 2 & 17974.7446 & 17974.7451 & -0.0005 \\
5 & 1 & 4 & 4 & 1 & 3 & 18552.5924 & 18552.5931 & -0.0007 \\
\hline
\end{tabular}

Table S17: syn-3-furfural $\left({ }^{13} \mathrm{C} 6\right)$

\begin{tabular}{ccccccccc}
\hline $\mathrm{J}^{\prime}$ & $\mathrm{K}_{\mathrm{a}}{ }^{\prime}$ & $\mathrm{K}_{\mathrm{c}}{ }^{\prime}$ & $\mathrm{J}^{\prime \prime}$ & $\mathrm{K}_{\mathrm{a}}{ }^{\prime \prime}$ & $\mathrm{K}_{\mathrm{c}^{\prime \prime}}$ & $v_{\text {obs }} / \mathrm{MHz}$ & $v_{\text {calc }} / \mathrm{MHz}$ & $v_{\text {obs }- \text { calc }} / \mathrm{MHz}$ \\
\hline 2 & 1 & 2 & 1 & 1 & 1 & 6711.5272 & 6711.5278 & -0.0005 \\
2 & 0 & 2 & 1 & 0 & 1 & 7076.4726 & 7076.4719 & 0.0007 \\
2 & 1 & 1 & 1 & 1 & 0 & 7475.8753 & 7475.8744 & 0.0008 \\
3 & 1 & 2 & 3 & 0 & 3 & 7574.1364 & 7574.1380 & -0.0015 \\
4 & 1 & 3 & 4 & 0 & 4 & 8472.7522 & 8472.7514 & 0.0007 \\
4 & 0 & 4 & 3 & 1 & 3 & 8735.5526 & 8735.5534 & -0.0007 \\
3 & 1 & 3 & 2 & 1 & 2 & 10056.7481 & 10056.7487 & -0.0005 \\
3 & 0 & 3 & 2 & 0 & 2 & 10571.8526 & 10571.8532 & -0.0006 \\
3 & 1 & 2 & 2 & 1 & 1 & 11202.7797 & 11202.7785 & 0.0012 \\
6 & 1 & 5 & 6 & 0 & 6 & 11244.6416 & 11244.6430 & -0.0013 \\
5 & 0 & 5 & 4 & 1 & 4 & 12745.2617 & 12745.2620 & -0.0002 \\
2 & 1 & 2 & 1 & 0 & 1 & 12873.1656 & 12873.1640 & 0.0015 \\
4 & 1 & 4 & 3 & 1 & 3 & 13389.9853 & 13389.9868 & -0.0015 \\
4 & 0 & 4 & 3 & 0 & 3 & 14017.1402 & 14017.1409 & -0.0007 \\
4 & 2 & 3 & 3 & 2 & 2 & 14173.9548 & 14173.9546 & 0.0001 \\
4 & 2 & 2 & 3 & 2 & 1 & 14344.1826 & 14344.1818 & 0.0008 \\
4 & 1 & 3 & 3 & 1 & 2 & 14915.7550 & 14915.7544 & 0.0006 \\
3 & 1 & 3 & 2 & 0 & 2 & 15853.4419 & 15853.4408 & 0.0011 \\
5 & 1 & 5 & 4 & 1 & 4 & 16708.3251 & 16708.3276 & -0.0024 \\
6 & 0 & 6 & 5 & 1 & 5 & 16750.0613 & 16750.0596 & 0.0016 \\
5 & 0 & 5 & 4 & 0 & 4 & 17399.6931 & 17399.6954 & -0.0022 \\
5 & 2 & 4 & 4 & 2 & 3 & 17695.8973 & 17695.8970 & 0.0002 \\
5 & 3 & 3 & 4 & 3 & 2 & 17788.4297 & 17788.4301 & -0.0003 \\
5 & 2 & 3 & 4 & 2 & 2 & 18030.3116 & 18030.3096 & 0.0019 \\
5 & 1 & 4 & 4 & 1 & 3 & 18608.9995 & 18608.9990 & 0.0005 \\
\hline
\end{tabular}

\section{Appendix IV: Kraitchman Coordinates $\left(r_{s}\right)$}

Table S18: $r_{s}$ coordinates for anti-3-furfural 


\begin{tabular}{|c|c|c|c|c|}
\hline \multicolumn{5}{|l|}{${ }^{18} \mathrm{O} 1$} \\
\hline & $\mathrm{a}$ & $\mathrm{b}$ & & \\
\hline PLANAR: & $1.93665+-0.00000$ & 0.47092 & +-0.00000 & \\
\hline +Costain err. & $\begin{array}{l}1.93665+-0.00077 \\
a\end{array}$ & $\begin{array}{l}0.47092 \\
b\end{array}$ & +-0.00319 & C \\
\hline NONPLANAR: & $1.93659+-0.00000$ & 0.47064 & +-0.00001 & $0.01620+-0.00021$ \\
\hline +Costain err. & $1.93659+-0.00077$ & 0.47064 & +-0.00319 & $0.01620+-0.09260$ \\
\hline \multicolumn{5}{|l|}{${ }^{13} \mathrm{C} 2$} \\
\hline & $\mathrm{a}$ & $\mathrm{b}$ & & \\
\hline PLANAR: & $0.73211+-0.00001$ & 1.09721 & +-0.00000 & \\
\hline +Costain err. & $\begin{array}{l}0.73211+-0.00205 \\
a\end{array}$ & $\begin{array}{l}1.09721 \\
b\end{array}$ & +-0.00137 & c \\
\hline NONPLANAR: & $0.73273+-0.00001$ & 1.09764 & +-0.00001 & $0.03054 * i+-0.00023$ \\
\hline +Costain err. & $0.73273+-0.00205$ & 1.09764 & +-0.00137 & $0.03054 * \mathrm{i}+-0.04912$ \\
\hline \multicolumn{5}{|l|}{${ }^{13} \mathrm{C} 3$} \\
\hline & a & $b$ & & \\
\hline PLANAR: & $0.25466+-0.00002$ & 0.18201 & +-0.00001 & \\
\hline +Costain err. & $\begin{array}{l}0.25466+-0.00589 \\
a\end{array}$ & $\begin{array}{l}0.18201 \\
b\end{array}$ & +-0.00824 & c \\
\hline NONPLANAR: & $0.25350+-0.00002$ & 0.18037 & +-0.00003 & $0.02437+-0.00020$ \\
\hline +Costain err. & $0.25350+-0.00592$ & 0.18037 & +-0.00832 & $0.02437+-0.06155$ \\
\hline \multicolumn{5}{|l|}{${ }^{13} \mathrm{C} 4$} \\
\hline & $\mathrm{a}$ & $\mathrm{b}$ & & \\
\hline PLANAR: & $0.33110+-0.00004$ & 1.10995 & +-0.00000 & \\
\hline +Costain err. & $\begin{array}{l}0.33110+-0.00453 \\
a\end{array}$ & $\begin{array}{l}1.10995 \\
b\end{array}$ & +- 0.00135 & c \\
\hline NONPLANAR: & $0.33180+-0.00003$ & 1.11017 & +-0.00001 & $0.02196 * i+-0.00052$ \\
\hline +Costain err. & $0.33180+-0.00452$ & 1.11017 & +-0.00135 & $0.02196 * i+-0.06831$ \\
\hline
\end{tabular}




$\begin{array}{llll} & \mathrm{a} & \mathrm{b} & \\ \text { PLANAR: } & 1.67610+-0.00000 & 0.87545+-0.00000 & \\ \text { +Costain err. } & 1.67610+-0.00089 & 0.87545+-0.00171 & \\ & \mathrm{a} & \mathrm{b} & \mathrm{c} \\ \text { NONPLANAR: } & 1.67621+-0.00000 & 0.87567+-0.00001 & 0.01958 * \mathrm{i}+-0.00038 \\ \text { +Costain err. } & 1.67621+-0.00089 & 0.87567+-0.00171 & 0.01958 * \mathrm{i}+-0.07660\end{array}$

$\begin{array}{llll}{ }^{13} \text { C6 } & & & \\ & & & \\ & \mathrm{a} & \mathrm{b} & \\ \text { PLANAR: } & 1.70649+0.00001 & 0.50230+-0.00000 & \\ \text { +Costain err. } & 1.70649+-0.00088 & 0.50230+-0.00299 & \\ & \mathrm{a} & \mathrm{b} & \mathrm{c} \\ \text { NONPLANAR: } & 1.70637+-0.00000 & 0.50189+-0.00001 & 0.02027+-0.00035 \\ \text { +Costain err. } & 1.70637+0.00088 & 0.50189+-0.00299 & 0.02027+-0.07401\end{array}$

\begin{tabular}{|c|c|c|c|}
\hline \multicolumn{4}{|l|}{${ }^{18} \mathrm{O} 7$} \\
\hline & a & $b$ & \\
\hline PLANAR: & $2.58544+-0.00000$ & $0.33559+-0.00000$ & \\
\hline +Costain err. & $2.58544+-0.00058$ & $0.33559+-0.00447$ & \\
\hline & a & $\mathrm{b}$ & c \\
\hline NONPLANAR: & $2.58545+-0.00000$ & $0.33571+-0.00001$ & $0.00909 * i+-0.00027$ \\
\hline +Costain err. & $2.58545+-0.00058$ & $0.33571+-0.00447$ & $0.00909 * i+-0.16508$ \\
\hline
\end{tabular}

Table S18: $r_{s}$ coordinates for syn-3-furfural

\begin{tabular}{|c|c|c|c|}
\hline \multicolumn{4}{|c|}{${ }^{13} \mathrm{C} 2$} \\
\hline & a & $\mathrm{b}$ & \\
\hline PLANAR: & $0.30398+-0.00003$ & $0.99300+-0.00000$ & \\
\hline +Costain err. & $\begin{array}{l}0.30398+-0.00493 \\
a\end{array}$ & $\begin{array}{l}0.99300+-0.00151 \\
b\end{array}$ & c \\
\hline NONPLANAR: & $0.30429+-0.00002$ & $0.99310+-0.00001$ & $0.01390 * i+-0.00051$ \\
\hline +Costain err. & $0.30429+-0.00493$ & $0.99310+-0.00151$ & $0.01390 * i+-0.10794$ \\
\hline
\end{tabular}




$\begin{array}{llll} & \mathrm{a} & \mathrm{b} & \\ \text { PLANAR: } & 0.24568+-0.00002 & 0.23252+-0.00001 & \\ \text { +Costain err. } & 0.24568+-0.00611 & 0.23252+-0.00645 & \\ & \mathrm{a} & \mathrm{b} & \mathrm{c} \\ \text { NONPLANAR: } & 0.24466+-0.00002 & 0.23144+-0.00002 & 0.02235+-0.00022 \\ \text { +Costain err. } & 0.24466+-0.00613 & 0.23144+-0.00648 & 0.02235+-0.06711\end{array}$

\begin{tabular}{|c|c|c|c|}
\hline & a & $b$ & \\
\hline PLANAR: & $0.79316+-0.00001$ & $1.19663+-0.00000$ & \\
\hline +Costain err. & $\begin{array}{l}0.79316+-0.00189 \\
a\end{array}$ & $\begin{array}{l}1.19663+-0.00125 \\
b\end{array}$ & c \\
\hline NONPLANAR: & $0.79383+-0.00001$ & $1.19710+-0.00001$ & $0.03336 * i+-0.00024$ \\
\hline +Costain err. & $0.79383+-0.00189$ & $1.19710+-0.00125$ & $0.03336 * i+-0.04497$ \\
\hline
\end{tabular}

\begin{tabular}{|c|c|c|c|}
\hline \multicolumn{4}{|l|}{${ }^{13} \mathrm{C} 5$} \\
\hline & $a$ & $b$ & \\
\hline PLANAR: & $1.94451+-0.00000$ & $0.47126+-0.00000$ & \\
\hline +Costain err. & $\begin{array}{l}1.94451+-0.00077 \\
a\end{array}$ & $\begin{array}{l}0.47126+-0.00318 \\
b\end{array}$ & c \\
\hline NONPLANAR: & $1.94442+-0.00000$ & $0.47087+-0.00001$ & $0.01911+-0.00032$ \\
\hline +Costain err. & $1.94442+-0.00077$ & $0.47087+-0.00319$ & $0.01911+-0.07851$ \\
\hline
\end{tabular}

\begin{tabular}{|c|c|c|c|}
\hline \multicolumn{4}{|l|}{${ }^{13} \mathrm{C} 6$} \\
\hline & $\mathrm{a}$ & $b$ & \\
\hline PLANAR: & $1.71504+-0.00001$ & $0.50278+-0.00001$ & \\
\hline +Costain err. & $\begin{array}{l}1.71504+-0.00087 \\
a\end{array}$ & $\begin{array}{l}0.50278+-0.00298 \\
b\end{array}$ & C \\
\hline NONPLANAR: & $1.71487+-0.00001$ & $0.50221+-0.00002$ & $0.02394+-0.00041$ \\
\hline +Costain err. & $1.71487+-0.00087$ & $0.50221+-0.00299$ & $0.02394+-0.06265$ \\
\hline
\end{tabular}

